\title{
TLAOLI PUMA, HÍBRIDO DE MAÍZ PARA GRANO Y FORRAJE CON ANDROESTERILIDAD Y RESTAURACIÓN DE LA FERTILIDAD MASCULINA
}

\author{
TLAOLI PUMA, A MAIZE HYBRID FOR GRAIN AND FORAGE WITH MALE \\ STERILITY AND RESTORATION OF MALE FERTILITY
}

\author{
Margarita Tadeo-Robledo', Alejandro Espinosa-Calderón² ${ }^{2}$ Job Zaragoza-Esparza', \\ Consuelo López-López ${ }^{1}$, Enrique I. Canales-Islas ${ }^{3}$, Benjamín Zamudio-González ${ }^{4}$, Antonio \\ Turrent-Fernández ${ }^{2}$, Juan Virgen-Vargas ${ }^{2 \dagger}$, Mauro Sierra-Macías ${ }^{5}$, Noel 0. Gómez- \\ Montiel $^{6}$, Karina Y. Mora-García ${ }^{1}$, Pablo Andres-Meza ${ }^{7}$ y Alma L. Cárdenas-Marcelo ${ }^{8}$
}

\begin{abstract}
'Universidad Nacional Autónoma de México, Facultad de Estudios Superiores Cuautitlán, Cuautitlán Izcalli, Estado de México, México. ${ }^{2}$ Instituto Nacional de Investigaciones Forestales, Agrícolas y Pecuarias (INIFAP), Campo Experimental Valle de México, Coatlinchán, Texcoco, Estado de México, México. ${ }^{3}$ NIFAP, Campo Experimental Santiago Ixcuintla, Santiago Ixcuintla, Nayarit, México. ${ }^{4}$ INIFAP, Campo Experimental Valle de México Sitio Toluca, Zinacantepec, Estado de México., México. ${ }^{5}$ NIFAP, Campo Experimental Cotaxtla, Medellín, Veracruz, México. ${ }^{6}$ INIFAP, Campo Experimental Iguala, Iguala, Guerrero, México. `Universidad Veracruzana, Amatlán de los Reyes, Veracruz, México. ${ }^{8}$ Universidad Autónoma del Estado de México, El Cerrillo Piedras Blancas, Toluca, Estado de México, México.
\end{abstract}

*Autor de correspondencia (espinoale@yahoo.com.mx)

México importó 18 millones de toneladas de grano de maíz amarillo (Zea mays L.) en promedio los últimos años (González, 2020; Com. Pers.) ${ }^{7}$. En México, el maíz es el cultivo más importante por superficie sembrada (7.4 a 7.7 millones de hectáreas) y por consumo, de $200 \mathrm{~kg}$ por persona por año; ante este escenario, es imperativo lograr suficiencia y soberanía alimentaria en maíz (US Grains Council, 2020). Por lo anterior, es urgente incrementar la producción de grano, para lo cual se requieren variedades mejoradas públicas con buena productividad (Luna et al., 2012; Martínez-Gutiérrez et al., 2018; Tadeo-Robledo et al., 2016).

En los Valles Altos de México (2200 a 2600 msnm) se cosechan más de dos millones de hectáreas de maíz con rendimiento promedio de $2.82 \mathrm{t} \mathrm{ha}^{-1}$ (Virgen-Vargas et al., 2016). Se plantea que con un mayor uso y oferta de variedades mejoradas se podrían elevar los rendimientos de maíz en 300 mil ha, en áreas con humedad residual, punta de riego y buen temporal, donde el rendimiento promedio es de $3.5 \mathrm{t} \mathrm{ha}^{-1}$ de grano. Este rendimiento podría incrementarse con el uso de híbridos de alto rendimiento que producen hasta $6.0 \mathrm{t} \mathrm{ha}^{-1}$ (Martínez-Gutiérrez et al., 2018; Tadeo-Robledo et al., 2016).

En los lotes de producción comercial de semilla híbrida de maíz, al progenitor femenino generalmente se le elimina la espiga para mantener la calidad genética de la semilla; esta práctica requiere de 24 a 50 jornales por hectárea,

'González G. S. (2020) México importará cifra récord de maíz este año por la sequía. La Jornada, Sección de Economía. 21 de enero de 2020, Ciudad de México. https://www.jornada.com. mx/ultimas/economia/2020/01/21/mexico-importara-cifrarecord-de-maiz-este-ano-por-la-sequia-8730.html (Mayo 2021)

Recibido: 8 de abril de 2020

Aceptado: 15 de enero de 2021 según la uniformidad de las plantas, presencia de tallos secundarios y facilidad para retirar la espiga (MartínezLázaro et al., 2005; Tadeo-Robledo et al., 2016). Una alternativa para eliminar el desespigamiento es el uso de progenitores androestériles a través de la androesterilidad génico-citoplásmica (Espinosa et al., 2019; TadeoRobledo et al., 2016), con ésto se facilita la producción de semilla, se reducen los costos y se permite ofertar semillas de híbridos a menor precio (Martínez-Lázaro et al., 2005; Tadeo et al., 2018).

Desde 1992, investigadores de la Facultad de Estudios Superiores Cuautitlán de la Universidad Nacional Autónoma de México (FESC UNAM) y del Instituto Nacional de Investigaciones Forestales, Agrícolas y Pecuarias (INIFAP) han trabajado con fuentes de esterilidad masculina en maíz, así como en la restauración de la fertilidad, incorporándola a los progenitores de híbridos ya existentes y en proceso de desarrollo, con el objeto de contar con híbridos que no requieran desespigamiento, utilicen androesterilidad y que dicha fertilidad sea restituida en las siembras comerciales (Espinosa et al., 2019; Tadeo-Robledo et al., 2016). Como producto de estos trabajos, se obtuvo el híbrido trilineal de maíz Tlaoli Puma; este híbrido fue inscrito en el Catálogo Nacional de Variedades Vegetales (CNVV) logrando su registro (3480-MAZ-1829-210617/C) en el año 2017; también obtuvo el título de obtentor número 2133, a favor de la UNAM el 27 de marzo de 2019. En este escrito se presenta información del híbrido Tlaoli Puma, sus características agronómicas, rendimiento y otras características específicas de uso para los productores de maíz de Valles Altos (Martínez-Gutiérrez et al., 2018). 
Tlaoli Puma es un híbrido trilineal de grano blanco y textura semidentada, con adaptación a Valles Altos (de 2200 a $2600 \mathrm{msnm}$ ). En sus progenitores se incorporó androesterilidad y también un macho restaurador de la fertilidad masculina para facilitar la producción de semillas, mantiene la calidad genética y apoya el abastecimiento de éstas por parte de empresas semilleras y grupos de productores. El híbrido Tlaoli Puma posee madurez intermedia, con floración masculina a los 87 dds y femenina a los 89 dds. Su rendimiento a través de varios años de evaluación (2008 a 2012) y localidades en el Estado de México (Cuautitlán, Ixtlahuaca, Texcoco, Temascalcingo y Atlacomulco) fue de $11.7 \mathrm{t} \mathrm{ha}^{-1}$, con variación de 8.7 a 13.5 t ha $^{-1}$; en promedio, el comportamiento es superior en 26.0 $\%$ con respecto al híbrido testigo $\mathrm{H}-48$, en uso comercial extensivo desde 1998. Tlaoli Puma es unos días más tardío que H-48, alcanza madurez fisiológica a los 166 dds, y posteriormente es posible cortar, "amogotar" (secado en campo) y efectuar la pizca manual; de ser el caso, la cosecha mecánica puede efectuarse a los 181 dds. La altura de la planta varía de 262 a $270 \mathrm{~cm}$ y la de mazorca de 127 a $140 \mathrm{~cm}$. La longitud de mazorca es de $18.5 \mathrm{~cm}$, con 16 hileras y 33 granos por hilera (Figura 1); su relación grano/mazorca es de $86 \%$. Es resistente al acame de raíz y tallo y a las enfermedades de planta y mazorca (Puccinia y Ustilago). Tlaoli Puma expresa buena productividad en ensilado, ya que su rendimiento de forraje es superior a 85 t ha- ${ }^{-1}$, producción de materia seca de $25 \mathrm{t} \mathrm{ha}^{-1}$ y porcentaje de digestibilidad de $67 \%$, por lo que se recomienda para doble propósito, grano y forraje. Al evaluar el grano para la elaboración de tortilla, Tlaoli Puma expresó un rendimiento de masa superior a lo requerido por los industriales de la masa y la tortilla (2.0 kg de masa $\mathrm{kg}^{-1}$ de maíz procesado), con peso volumétrico de $76 \mathrm{~kg} \mathrm{hL}^{-1}$ e índice de flotación de $14 \%$.

La estructura genética del híbrido es (IA442AEC $\times$ IA446) × IA449RMIA44; en su cruza trilineal se emplea la cruza androestéril IA442AEC $\times$ IA446, en la cual la línea IA442AEC androestéril, y tiene su versión isogénica fértil en la línea IA442 F; esta línea se obtuvo de un compuesto de amplia base genética, a partir de selección a través de autofecundaciones, cruzamientos planta a planta, autofecundaciones y ciclos de avance fraternal. La línea IA442AEC fue desarrollada en la UNAM, al incorporarle a la línea IA442 F la androesterilidad tipo C, la cual está identificada en la UNAM con la genealogía CXP3PL7, caracterizada por su estabilidad y acción en el grupo C.

La línea IA442 F fue utilizada como progenitor masculino, para cruzarse con la fuente de androesterilidad; posteriormente, se efectuaron seis retrocruzas hacia la línea IA442 F, para obtener la versión de esterilidad masculina de la línea IA442 AEC, la cual se considera la versión isogénica, cuya mantenedora es la propia línea IA442 F en su versión fértil. IA446 es una línea de la raza Cónico, derivada y avanzada en la UNAM a partir de la $\mathrm{F}_{2}$ de un híbrido obtenido en la propia UNAM. La línea IA449RMIA44 es derivada y avanzada de la cruza de la línea IA449, ya registrada por la UNAM, con la línea MIA44, que tiene el carácter de capacidad de restauración de la fertilidad masculina, propiedad de la UNAM. Después de verificar la capacidad restauradora se obtuvieron dos ciclos de recombinación fraternal; posteriormente, se avanzó durante seis ciclos de autofecundación. Para garantizar la pureza genética de Tlaoli Puma, la semilla de este híbrido trilíneal y sus progenitores (tres líneas, cruza simple e híbrido trilineal) deben incrementarse en lotes aislados separados al menos $300 \mathrm{~m}$ para semilla básica (líneas) y registrada (cruza simple) y $200 \mathrm{~m}$ para multiplicar semilla certificada; también es posible aislar el lote mediante fechas de siembra con una diferencia de 20 días, de tal forma que no haya coincidencia de la floración del lote de producción de semilla de Tlaoli Puma con otros lotes. Deben eliminarse las plantas fuera de tipo y enfermas, por lo menos en tres etapas, a los 25 y 50 días y previo a antesis.

Al momento de la cosecha puede mejorarse aún la calidad del lote de semillas eliminando las mazorcas fuera de tipo, mal formadas o con algún grado de pudrición o daño. Para la renovación y mantenimiento de la semilla original del híbrido Tlaoli Puma, la línea IA442 F debe incrementarse por autofecundaciónes. La línea IA442 AEC es androestéril y conserva su identidad combinándose con la línea IA442F, la cual es su mantenedora isogénica fértil. La línea IA446 se incrementa a través de autofecundaciones o en forma fraternal. La línea IA449RMIA44 debe incrementarse por cruzamientos fraternales o en cadena. La cruza simple hembra, versión androestéril del híbrido trilíneal, conserva su identidad varietal al cruzar las líneas IA442AEC $\times$ IA446. El híbrido trilíneal en campo se obtiene usando la conformación (IA442AEC × IA446) × IA449RMIA44, dado que la línea IA449RMIA44 posee capacidad restauradora de la fertilidad masculina, la semilla resultante es el híbrido trilíneal con fertilidad completa, no requiriéndose ninguna otra versión ni mezclas de semilla androestéril y fértil.

El híbrido comercial Tlaoli Puma en terrenos de agricultores es $100 \%$ fértil; las prácticas agronómicas para conservar las características que identifican al híbrido deben apegarse a las recomendaciones para producir semilla de este tipo de híbridos, desmezclar en tres oportunidades y apegarse a la descripción. El tamaño de la población para el incremento de cada línea requiere como mínimo de 100 plantas por incremento para conservar las características que identifican a cada línea. Para producción de semilla híbrida debe limitarse el número 


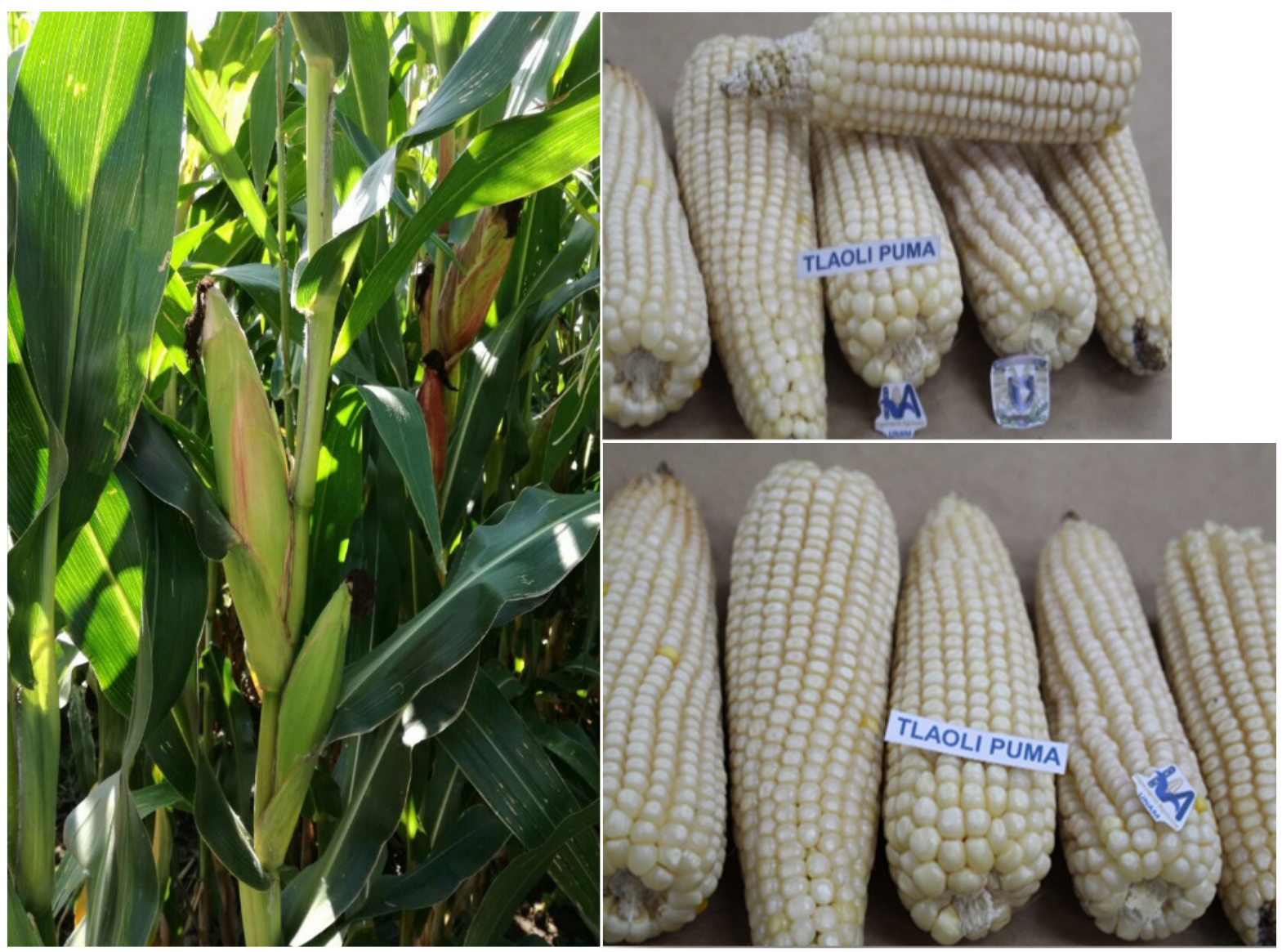

Figura 1. Planta y mazorca del híbrido de maíz Tlaoli Puma.

de generaciones a la básica para la línea IA449RMIA44 y registrada para la cruza simple IA442AEC $\times$ IA446. La semilla registrada de los progenitores de Tlaoli Puma está disponible en la FESC UNAM para incrementar semilla certificada por interesados.

\section{AGRADECIMIENTOS}

Esta investigación fue financiada por el Programa de Apoyo a Proyectos de Investigación e Innovación Tecnológica (PAPIIT) de la Dirección General del Personal Académico de la UNAM, clave: IT201618.

\section{BIBLIOGRAFÍA}

Espinosa C. A., M. Tadeo R., J. Virgen V., B. Zamudio G., A. Turrent F. y C. López L. (2019) H 47AE, H 49AE y H 53AE híbridos de maíz con androesterilidad para Valles Altos. Primer Simposio para la Autosuficiencia y Soberanía Alimentaria de México. V Reunión Estatal de Investigación. Publicación Especial Núm. 1, Campo Experimental Valle de México, INIFAP. Texcoco, Estado de México. pp:8-12.

Luna M. B. M., M. A. Hinojosa R., O. J. Ayala G., F. Castillo G. y J. A. Mejía C. (2012) Perspectivas de desarrollo de la industria semillera de maíz en México. Revista Fitotecnia Mexicana 35:1-7, https:// doi.org/10.35196/rfm.2012.1.1

Martínez-Gutiérrez A., B. Zamudio-González, M. Tadeo-Robledo, A. EspinosaCalderón, J. C. Cardoso-Galvão, G. Vázquez-Carrillo y A. TurrentFernández (2018) Rendimiento de híbridos de maíz grano blanco en cinco localidades de Valles Altos de México. Revista Mexicana de Ciencias Agrícolas 9:1447-1458, https://doi. org/10.29312/remexca.v9i7.1357

Martínez-Lázaro C., L. E. Mendoza-Onofre, G. García-de los Santos, M. C Mendoza-Castillo y A. Martínez-Garza (2005) Producción de semilla híbrida de maíz con líneas androestériles y androestérilesisogénicas y su respuesta a la fertilización y densidad de población. Revista Fitotecnia Mexicana 28:127-133.

Tadeo R. M., A. Espinosa C., J. J. García Z., R. Lobato O., N. O. Gómez M., M. Sierra M., ... y B. Zamudio-González (2018) Productivity of three maize hybrids under different proportions of male sterile and fertile seeds. Interciencia 43:852-857.

Tadeo-Robledo M., A. Espinosa-Calderón, J. J. García-Zavala, R. Lobato-Ortiz, N. O. Gómez-Montiel, M. Sierra-Macías, ... y H. J. Alcántar-Lugo (2016) Tsiri Puma, híbrido de maíz para Valles Altos con esquema de androesterilidad para producción de semillas. Revista Fitotecnia Mexicana 39:331-333, https://doi.org/10.35196/ rfm.2016.3.331-333

US Grains Council (2020) Informe de la calidad de la cosecha de maíz 2019/2020. US Grains Council. Washington, D. C. https://grains. org/lta/resources-page/reports/reportes-de-calidad-de-maiz/ (Junio 2021)

Virgen-Vargas J., R. Zepeda-Bautista, M. A. Ávila-Perches, I. Rojas-Martínez, A. Espinosa-Calderón y A. J. Gámez-Vázquez (2016) Desespigamiento en cruzas simples progenitoras de híbridos de maíz (Zea mays L.) para Valles Altos de México. Agrociencia 50:43-59. 
